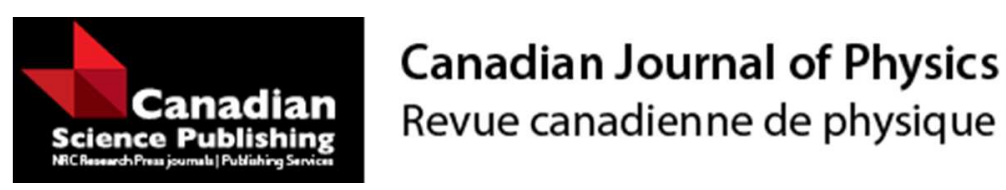

\title{
Laser control on molecular harmonic emission and isolated attosecond pulse generation
}

\begin{tabular}{|r|l|}
\hline Journal: & Canadian Journal of Physics \\
\hline Manuscript ID & cjp-2016-0774.R1 \\
\hline Manuscript Type: & Article \\
\hline Complete List of Authors: & $\begin{array}{l}\text { Liu, Hang; Liaoning University of Technology, } \\
\text { Feng, Rick; Liaoning University of Technology, School of Chemical and } \\
\text { Environmental Engineering }\end{array}$ \\
\hline Keyword: & $\begin{array}{l}\text { molecular high-order harmonic generation, laser control, distributions of } \\
\text { harmonic spectra, half-cycle controlling pulse, isolated attosecond pulse }\end{array}$ \\
\hline \multicolumn{2}{|c}{} \\
\hline
\end{tabular}




\title{
Laser control on molecular harmonic emission and isolated attosecond pulse generation
}

Hang Liu* and Rick Feng

\begin{abstract}
Laser control on the generations of the molecular high-order harmonic generation (MHHG) and the isolated attosecond pulse (IAP) from $\mathrm{H}_{2}^{+}$have been theoretically investigated through solving the Non-Bohn-Oppenheimer (NBO) time-dependent Schrödinger equation (TDSE). It is found that (i) in the middle laser intensity (i.e. $I=4.0 \times 10^{14} \mathrm{~W} / \mathrm{cm}^{2}$ ), the contribution from negative-H to the MHHG is higher than that from the positive-H. With the decrease (i.e. $I=2.0 \times 10^{14} \mathrm{~W} / \mathrm{cm}^{2}$ ) or the increase (i.e. $I=7.0 \times 10^{14} \mathrm{~W} / \mathrm{cm}^{2}$ ) in laser intensity, the asymmetric contributions from the two $\mathrm{H}$ nuclei to the MHHG are decreased. (ii) Pulse duration investigation shows that the distributions of the MHHG in two $\mathrm{H}$ nuclei present the similar contributions as the pulse duration enhanced. (iii) Laser phase investigation shows that when the laser phase is chosen from $0.0 \pi$ to $0.6 \pi$ and from $1.7 \pi$ to $2.0 \pi$, the contribution from the negative- $H$ plays the main role in MHHG. When the laser phase is chosen from $0.7 \pi$ to the $1.6 \pi$, the contribution from the positive- $\mathrm{H}$ to the MHHG is remarkably enhanced and becomes greater than that from the negative-H. The time-frequency analyses of the MHHG and
\end{abstract}

\footnotetext{
Hang Liu. School of Chemical and Environmental Engineering, Liaoning University of Technology, Jinzhou, 121000, China.

Rick Feng. School of Chemical and Environmental Engineering, Liaoning University of Technology, Jinzhou, 121000, China.

* Corresponding author Hang Liu (Email: attophys_lngy@126.com)
} 
the time-dependent wave function are shown to explain harmonic emission process and the electron motion in $\mathrm{H}_{2}{ }^{+}$. (iv) With the introduction of one or two half-cycle controlling pulses, the cutoff and the intensity of the MHHG spectra can be controlled. Finally, by selecting the harmonics generated during single harmonic emission event, an IAP with the full width at half maximum (FWHM) of 48 as can be produced.

Keywords: molecular high-order harmonic generation; laser control; distributions of harmonic spectra; half-cycle controlling pulse; isolated attosecond pulse PACS Nos.: 42.65.Ky, 42.65.Re, 32.80.Fb

\section{INTRODUCTION}

With the development of the laser technology and the computational method, the interaction between the intense laser pulse $\left(I>10^{13} \mathrm{~W} / \mathrm{cm}^{2}\right)$ and the smaller molecules (i.e. $\mathrm{H}_{2}{ }^{+}, \mathrm{HeH}^{2+}, \mathrm{H}_{2}, \mathrm{HeH}^{+}$etc) attracts lots of attention in optical physics [1, 2] and leads to many new interesting phenomena. Such as, the bond softening and hardening $[3,4]$; the asymmetric ionization and dissociation channels $[5,6]$; the charge-resonance-enhanced-ionization $[7,8]$; the dissociative ionization [9], the kinetic-energy spectra of the dissociation and the Coulomb explosion channels [10]; the above-threshold dissociation (ATD) and the above-threshold ionization (ATI) $[11,12]$; the molecular high-order harmonic generation (MHHG) [13-26].

Among them, MHHG as the most important and potential tool to obtain the coherent $\mathrm{XUV}$ and X-ray pulses and to explore the electron dynamics in molecules has been 
extensively investigated during the last two decades. For instance, Lein [13], Feng et al. [14] and Yu et al. [15] find that the MHHG is relevant to the initial vibrational state and the harmonic intensity can be enhanced with the increase of the initial vibrational state. Yuan and Bandrauk $[16,17]$ theoretically investigated the generations of the circularly or elliptically polarized harmonics in $\mathrm{H}_{2}^{+}$and obtained an isolated circularly polarized attosecond pulse with the combination of a few cycle elliptically polarized laser pulse and a terahertz field. Bian et al. [18], Miao et al. [19], Feng et al. [20] and Chen et al. [21] theoretically investigate the multi-channel harmonic emission from the asymmetric molecule $\mathrm{HeH}^{2+}$. Lan et al. [22] investigate to the destructive interference structure on the MHHG. Liu et al. [23] theoretically investigate the selection rules of MHHG. Yavuz et al. [24] and Feng [25, 26] find that the plasmonic enhancement of the MHHG is entirely possible for the molecules.

As we know that the harmonic generation can be understood through the semi-classical model by Corkum [27] with the steps of (i) ionization, (ii) acceleration, and (iii) recombination. However, the molecules have more than one nucleus, thus the electron not only can recombine with its parent nucleus, but also with the others. Thus, currently, the distributions of the different nuclei to the MHHG become a subject of interesting issue. For example, Pei et al. [28] theoretically investigate the contributions of the two $\mathrm{H}$ nuclei to the MHHG in $\mathrm{H}_{2}{ }^{+}$. Zhang et al. [29-31] theoretically investigate and find that by properly adding the controlling pulse, the distributions of the MHHG in $\mathrm{H}_{2}^{+}$ can be controlled and the recombination process from the negative- $\mathrm{H}$ can be enhanced. Du et al. [32] theoretically investigate the distributions of the MHHG in $\mathrm{N}_{2}$ driven by the 
orthogonally polarized two-color field.

Thus, in this paper, we investigate this interesting issue of controlling the distributions of MHHG and the isolated attosecond pulse generation (IAP) in $\mathrm{H}_{2}^{+}$. Moreover, through analyzing the time-frequency analyses of the MHHG and the time-dependent wave function, the attosecond-resolution electron dynamics in $\mathrm{H}_{2}{ }^{+}$and the reason for the distributions of the MHHG have been given.

\section{METHODS}

Non-Bohn-Oppenheimer time-dependent Schrödinger equation (NBO-TDSE) for $\mathrm{H}_{2}^{+}$can be expressed as [33-35],

$$
i \frac{\partial \psi(z, R, t)}{\partial t}=\left[-\frac{1}{2 u_{e}} \frac{\partial^{2}}{\partial z^{2}}-\frac{1}{2 u_{N}} \frac{\partial^{2}}{\partial R^{2}}+V(z, R)+\left(1+\frac{1}{\left(2 m_{H}+1\right)}\right) z E(t)\right] \psi(z, R, t) .
$$

$m_{H}, u_{N}=m_{H}^{2} /\left(2 m_{H}\right), u_{e}=2 m_{H} /\left(2 m_{H}+1\right)$ are the mass of $\mathrm{H}$ nucleus, and the reduced masses of the electron and the nucleus, respectively. $R$ and $z$ denote the nuclear and the electronic coordinates. $V(z, R)=1 / R-1 / \sqrt{(z-R / 2)^{2}+1.0}-1 / \sqrt{(z+R / 2)^{2}+1.0}$ is the Coulomb potential of $\mathrm{H}_{2}{ }^{+}$. The initial wave function can be expressed as $\psi_{\text {initial }}(z, R)=\phi(R) \chi(z, R)$, where $\phi(R)$ and $\chi(z, R)$ are the wave functions of the initial vibrational state and the ground electronic state, respectively. In the present paper, $\phi(R)$ and $\chi(z, R)$ are calculated from the eigenvalue equations,

$$
\begin{aligned}
& {\left[-\frac{1}{2 u_{e}} \frac{\partial^{2}}{\partial z^{2}}+V_{e}(z, R)\right] \chi(z, R)=E_{e}(R) \chi(z, R),} \\
& {\left[-\frac{1}{2 u_{N}} \frac{\partial^{2}}{\partial R^{2}}+E_{e}(R)+V_{n}(R)\right] \phi(R)=E \phi(R) .}
\end{aligned}
$$

The laser field is $E(t)=E \exp \left[-4 \ln (2) t^{2} / \tau^{2}\right] \sin \left(\omega_{1} t+\varphi\right)+E_{H C P}(t)$, where $\omega_{1}, E, \tau$ 
and $\varphi$ are the frequency, the amplitude, the pulse duration and the carrier envelope phase (CEP) of the 5 fs $/ 800 \quad$ nm $E_{H C P}(t)=k \theta\left(t-\tau_{\text {delay }}\right) E\left[\frac{400\left(t-\tau_{\text {delay }}\right)^{3} \exp \left[-8\left(t-\tau_{\text {delay }}\right) / \tau_{H C P}\right]}{\tau_{H C P}^{3}}\right.$
$\left.-\frac{0.004\left(t-\tau_{\text {delay }}\right)^{5} \exp \left[-\left(t-\tau_{\text {delay }}\right) / \tau_{H C P}\right]}{\tau_{H C P}^{5}}\right]$ half-cycle pulse [36] with pulse intensity $k E=0.3 E$, and pulse duration $\tau_{\mathrm{HCP}}=2.0$ fs. $\tau_{\text {delay }}$ is the delay time between the $800 \mathrm{~nm}$ field and the controlling half-cycle pulse.

Time-dependent induced dipole moment can be achieved by [37],

$$
\begin{aligned}
& d_{A-z<0}(t)=\int_{-\infty}^{0} \psi^{*}(z, R, t)\left(-\frac{\partial V(z, R)}{\partial z}+E(t)\right) \psi(z, R, t) d z, \\
& d_{A-z>0}(t)=\int_{0}^{\infty} \psi^{*}(z, R, t)\left(-\frac{\partial V(z, R)}{\partial z}+E(t)\right) \psi(z, R, t) d z,
\end{aligned}
$$

where $d_{A}(t)=d_{A-z<0}(t)+d_{A-z>0}(t)$.

MHHG spectra can be expressed as,

$$
\begin{aligned}
& S_{z<0}(\omega)=\left|\frac{1}{\sqrt{2 \pi}} \int_{0}^{T_{\text {total }}} \mathrm{d}_{A-z<0}(t) e^{-i \omega t} d t\right|^{2}, \\
& S_{z>0}(\omega)=\left|\frac{1}{\sqrt{2 \pi}} \int_{0}^{T_{\text {total }}} \mathrm{d}_{A-z>0}(t) e^{-i \omega t} d t\right|^{2},
\end{aligned}
$$

where $S(\omega)=S_{z<0}(\omega)+S_{z>0}(\omega)$.

Time-frequency analyses of the MHHG can be expressed as [38],

$$
\begin{aligned}
& A_{z<0}(t, \omega)=\int \mathrm{d}_{A-z<0}\left(t^{\prime}\right) \sqrt{\omega} W\left(\omega\left(t^{\prime}-t\right)\right) d t^{\prime}, \\
& A_{z>0}(t, \omega)=\int \mathrm{d}_{A-z>0}\left(t^{\prime}\right) \sqrt{\omega} W\left(\omega\left(t^{\prime}-t\right)\right) d t^{\prime} .
\end{aligned}
$$

where $W(x)=\left(\frac{1}{\sqrt{\xi}}\right) e^{i x} e^{-x^{2} / 2 \xi^{2}}$ with the wavelet window function fixed at $\xi=18$ a.u. which is about $1 / 6$ optical cycle of the fundamental $800 \mathrm{~nm}$ field. 
The IAP is obtained through the Fourier transformation of the harmonic continuum,

$$
I(t)=\left|\sum_{q}\left(\int \mathrm{d}_{A}(t) e^{-i q \omega t} d t\right) e^{i q \omega t}\right|^{2} .
$$

\section{RESULTS AND DISCUSSION}

\subsection{Attosecond-resolution molecular harmonic emission}

Figs. 1(a)-(c) show the distributions of the MHHG from $\mathrm{H}_{2}{ }^{+}$under the $5 \mathrm{fs} / 800 \mathrm{~nm}$ field with laser phase chosen to be $\varphi=0.0 \pi$. The laser intensities are $I=2.0 \times 10^{14} \mathrm{~W} / \mathrm{cm}^{2}$, $I=4.0 \times 10^{14} \mathrm{~W} / \mathrm{cm}^{2}, I=7.0 \times 10^{14} \mathrm{~W} / \mathrm{cm}^{2}$, respectively. It is found that (i) in the middle laser intensity (i.e. $I=4.0 \times 10^{14} \mathrm{~W} / \mathrm{cm}^{2}$ ), the contribution from negative-H to the MHHG is higher than that from the positive-H. With the decrease (i.e. $I=2.0 \times 10^{14} \mathrm{~W} / \mathrm{cm}^{2}$ ) or the increase (i.e. $I=7.0 \times 10^{14} \mathrm{~W} / \mathrm{cm}^{2}$ ) in laser intensity, the two $\mathrm{H}$ nuclei present the similar contributions to the MHHG.

To better understand the emission process of the MHHG, in Fig. 2, we present the profiles of the laser fields and the time-frequency analyses of the MHHG driven by the above fields. Clearly, there are three main harmonic emission events during the MHHG, marked as $A_{1}, A_{2}, A_{3}$ [Figs. 2(b), 2(c) for $I=2.0 \times 10^{14} \mathrm{~W} / \mathrm{cm}^{2}$; Figs. 2(e), 2(f) for $I=$ $4.0 \times 10^{14} \mathrm{~W} / \mathrm{cm}^{2}$; Figs. 2(h), 2(i) for $\left.I=7.0 \times 10^{14} \mathrm{~W} / \mathrm{cm}^{2}\right]$. According to the three-step model, each harmonic emission event is caused by the process of the ionization-acceleration-recombination. Through analyzing the profiles of the laser fields and the ionization probabilities (IPs) shown in Figs. 2(a), 2(d), 2(g) for the above three laser intensities, the ionizations happen near the peaks of the temporal amplitudes of laser 
field, marked as, $I_{1}, I_{2}, I_{3}$. After ionization, the electron can be accelerated in the following half-cycle, which is responsible for the above three harmonic emission events. Moreover, when the laser field is smaller than zero $(E(t)<0.0)$, the MHHG from the positive-H ( $\mathrm{z}>0.0$ a.u.) is higher than that from the negative-H $\left(\mathrm{z}<0.0\right.$ a.u.), i.e. $A_{1}$; while when the laser field changes its direction $(E(t)>0.0)$, the MHHG from the negative-H is larger than that from the positive-H (i.e. $\left.A_{2}\right)$. However, in the lower $(I=$ $\left.2.0 \times 10^{14} \mathrm{~W} / \mathrm{cm}^{2}\right)$ and higher $\left(I=7.0 \times 10^{14} \mathrm{~W} / \mathrm{cm}^{2}\right)$ laser intensities, the asymmetric distributions of the MHHG from the negative-H and positive-H are smaller than that in the middle laser intensity $\left(I=4.0 \times 10^{14} \mathrm{~W} / \mathrm{cm}^{2}\right)$. This is the reason behind the asymmetric distributions of the MHHG. It is noted that there is another harmonic emission event $A_{0}$ in the higher laser intensity $\left(7.0 \times 10^{14} \mathrm{~W} / \mathrm{cm}^{2}\right)$, which is caused by the former ionization-acceleration-recombination process from the $1.5 \mathrm{~T}$ to the $2.0 \mathrm{~T}$ ( $\mathrm{T}$ is the optical cycle of $800 \mathrm{~nm}$ field). However, the intensity of this harmonic emission process is too weak in comparison with the others. Thus, in this paper, we do not consider its contribution to the MHHG spectra.

Fig. 3 shows the distributions of the MHHG from $\mathrm{H}_{2}{ }^{+}$driven by the $10 \mathrm{fs} / 800 \mathrm{~nm}$ field with the laser intensity and laser phase chosen to be $I=4.0 \times 10^{14} \mathrm{~W} / \mathrm{cm}^{2}$ and $\varphi=$ $0.0 \pi$. Clearly, with the increase of the pulse duration, the asymmetric distributions of the MHHG from the two-H nuclei are decreased.

Fig. 4 shows the laser field and the time-frequency analyses of the MHHG driven by the $10 \mathrm{fs} / 800 \mathrm{~nm}$ field. As seen, as the pulse duration increased, the ionization points near the peaks of the temporal amplitudes of the half-cycle are increased (i.e. $I_{1} \sim I_{7}$ ), as shown 
in Fig. 4(a). As a result, the harmonic emission events are also increased, marked as $A_{1} \sim A_{7}$. Moreover, when the laser field is smaller $(E(t)<0.0)$ or larger $(E(t)>0.0)$ than zero, the MHHG from the positive-H is higher (i.e. $A_{1}$ and $A_{3}$ ) or smaller (i.e. $A_{2}$ and $A_{4}$ ) than that from the negative-H, respectively, as shown in Figs. 4(b) and 4(c). However, due to the harmonic emission events are increased, the MHHG from the two H nuclei present the similar contributions.

Fig. 5(a) shows laser phase effect on the distributions of the MHHG from the two-H nuclei (the harmonic intensities are integrated over the last 10 orders). The laser field is 5 fs/800 nm field with $I=4.0 \times 10^{14} \mathrm{~W} / \mathrm{cm}^{2}$. As seen, a periodic variation of the asymmetric distributions of the MHHG can be obtained during the region of $2 \pi$. In particular, (i) when the laser phase is chosen from $0.0 \pi$ to $0.6 \pi$ and from $1.7 \pi$ to $2.0 \pi$, the harmonic intensities from the negative- $\mathrm{H}$ are higher than those from the positive- $\mathrm{H}$. (ii) When the laser phase is chosen from $0.7 \pi$ to the $1.6 \pi$, the harmonic intensities from the positive- $\mathrm{H}$ are remarkably enhanced and become higher than those from the negative-H. And the maximum asymmetric harmonic intensities are achieved at $\varphi=0.0 \pi, 1.0 \pi, 2.0 \pi$, as shown in Fig. 5(b).

Fig. 6 shows the laser field and the time-frequency analyses of the MHHG driven by the above $5 \mathrm{fs} / 800 \mathrm{~nm}$ field with $\varphi=0.5 \pi$ and $1.0 \pi$. Comparing to Figs. $1(\mathrm{~b})$ and 2(d)-2(f), there are four and three main ionization points (corresponding to four and three harmonic emission events) for the cases of $\varphi=0.5 \pi$ and $\varphi=1.0 \pi$, respectively [see Figs. 6(a)-6(c) and 6(d)-6(f)]. Moreover, for the case of $\varphi=0.5 \pi$, the intensity of $A_{1}$ from the positive- $\mathrm{H}$ is higher than that from the negative- $\mathrm{H}$; and the intensity of $A_{2}$ from the 
negative- $\mathrm{H}$ is higher than that from the positive- $\mathrm{H}$; and the intensities of $A_{3}$ and $A_{4}$ present the similar contributions from the two nuclei, as shown in Figs. 6(b) and 6(c). For the case of $\varphi=1.0 \pi$, due to the laser profile is opposite to the case of $\varphi=0.0 \pi$ [see Figs. 2(d) and 6(d)], the intensities of the harmonic emission events $A_{1}-A_{3}$ present the opposite contributions in comparison with the case of $\varphi=0.0 \pi$. These are the reasons behind the asymmetric distributions of the MHHG.

Fig 7 shows the time-dependent wave function $|\psi(z, R, t)|^{2}$ of $\mathrm{H}_{2}{ }^{+}$driven by above $5 \mathrm{fs} / 800 \mathrm{~nm}$ field with $\varphi=0.0 \pi$. It is found that the electron prefers to localize on the negative-H at $t=2.0 \mathrm{~T}(E(t)>0.0)$. After half cycle, the electron transfers to the positive-H at $t=2.5 \mathrm{~T}(E(t)<0.0)$. With further acceleration in the laser field, the electron can transfer to the negative-H $[(E(t)>0.0)]$ and the positive- $\mathrm{H}[(E(t)<0.0)]$ at every half cycle. Through analyzing the time-frequency analyses of the MHHG and the time-dependent wave function, we see that the electron is more localized on the negative-H when the laser field is larger than zero $(E(t)>0.0)$; and the electron prefers to localize on the positive-H when the laser field changes its direction $(E(t)<0.0)$.

Fig. 8 shows the dressed Coulomb potential of $\mathrm{H}_{2}{ }^{+}$driven by the above $5 \mathrm{fs} / 800 \mathrm{~nm}$ field with $\varphi=0.0 \pi$. It shows that due to the values of the laser field are larger than zero at $t=2.0 \mathrm{~T}$ and $t=3.0 \mathrm{~T}$, the potential well of the negative-H are decreased, thus leading to the increase of the ionization from the negative-H. However, at $t=2.5 \mathrm{~T}$ and $t=3.5 \mathrm{~T}$, the values of the laser field are smaller than zero, thus resulting in the decrease of the potential well around the positive- $\mathrm{H}$ and is responsible for the electron transfer from the negative-H to the positive-H. Clearly, the dressed Coulomb potential caused by the 
temporal profile of the laser field is the main reason behind the electronic transfer between the two $\mathrm{H}$ nuclei.

\subsection{Single half-cycle pulse control on the molecular harmonic emission}

Figs. 9(a)-9(c) show the profiles of the combined fields driven by the above $5 \mathrm{fs} / 800$ $\mathrm{nm}$ field $\left(I=4.0 \times 10^{14} \mathrm{~W} / \mathrm{cm}^{2}, \varphi=0.0 \pi\right)$ and a half-cycle controlling pulse with $k E=0.3 E$, $\tau_{\mathrm{HCP}}=2.0$ fs and $\tau_{\text {delay }}=2.0 \mathrm{~T} ; 2.5 \mathrm{~T} ; 3.0 \mathrm{~T}$. As seen, with the introduction of the controlling half-cycle pulse, the temporal amplitudes of laser field from the $2.0 \mathrm{~T}$ to the $2.5 \mathrm{~T}$; from the $3.0 \mathrm{~T}$ to the $3.5 \mathrm{~T}$ and from the $2.5 \mathrm{~T}$ to the $3.0 \mathrm{~T}$ are decreased and increased, respectively. Figs. 9(d)-9(f) show the distributions of the MHHG under the above three combined fields. Firstly, we see that the contribution from the negative-H to the harmonic spectrum plays the main role in MHHG. Secondly, for the case of $\tau_{\text {delay }}=2.0 \mathrm{~T}$ [Fig. 9(d)], the intensity of the effective harmonic cutoff $\left(E_{\text {cutoff }}=65 \omega_{1}\right)$ is remarkably decreased in comparison with the single $800 \mathrm{~nm}$ field. For the case of $\tau_{\text {delay }}=2.5 \mathrm{~T}[$ Fig. $9(\mathrm{e})]$, the harmonic cutoff is extended in comparison with the single $800 \mathrm{~nm}$ field. For the case of $\tau_{\text {delay }}=3.0 \mathrm{~T}$ [Fig. 9(f)], the first harmonic cutoff is reduced, and the second harmonic plateau is broadened in comparison with the single $800 \mathrm{~nm}$ field.

Time-frequency analyses of the MHHG for the above three cases are shown in Fig. 10. For the case of $\tau_{\text {delay }}=2.0 \mathrm{~T}$ [Figs. $10(\mathrm{a})$ and $10(\mathrm{~b})$ ], due to the decrease of the temporal amplitude from $2.0 \mathrm{~T}$ to the $2.5 \mathrm{~T}$, the recombination energy of the harmonic emission event $A_{1}$ is decreased. As a result, there is only one harmonic emission event $A_{2}$ is responsible for the harmonic energy higher than $40 \omega_{1}$ and is the reason for the 
decrease of the harmonic intensity in the second harmonic plateau. For the case of $\tau_{\text {delay }}=$ 2.5T [Figs. 10(c) and 10(d)], the recombination energy of the harmonic emission event $A_{2}$ is remarkably enhanced compared with the single $800 \mathrm{~nm}$ field. This is because that the temporal amplitude of laser field from the $2.5 \mathrm{~T}$ to the $3.0 \mathrm{~T}$ is enhanced, thus, when the electron is ionized around the $2.25 \mathrm{~T}$, it can obtained much more recombination energy during the acceleration process from the $2.5 \mathrm{~T}$ to the $3.0 \mathrm{~T}$. For the case of $\tau_{\text {delay }}=3.0 \mathrm{~T}$ [Figs. 10(e) and 10(f)], the harmonic emission event $A_{3}$ is remarkably decreased in the presence of the combined field, which is responsible for the decrease of the first harmonic cutoff. This is attributing to the reduced amplitude of laser field from the 3.0T to the $3.5 \mathrm{~T}$, the ionized electron cannot receive the higher recombination energy during the acceleration process.

\subsection{Two half-cycle pulses control on the molecular harmonic emission}

Figs. 11(a) and 11(b) show the profiles of the combined fields driven by the above field and two half-cycle controlling pulses with $k E=0.3 E, \tau_{\mathrm{HCP}}=2.0$ fs and $\tau_{\text {delayl }}=2.0 \mathrm{~T}$, $\tau_{\text {delay } 2}=2.5 \mathrm{~T} ; \tau_{\text {delay } 1}=2.5 \mathrm{~T}, \tau_{\text {delay } 2}=3.0 \mathrm{~T}$. Clearly, by adding the two half-cycle pulses with $\tau_{\text {delay } 1}=2.0 \mathrm{~T}, \tau_{\text {delay } 2}=2.5 \mathrm{~T}$, the temporal amplitudes of laser field from the $2.0 \mathrm{~T}$ to the 2.5T; from the $2.5 \mathrm{~T}$ to the $3.0 \mathrm{~T}$ are decreased and increased; while with the introduction of the two half-cycle pulses with $\tau_{\text {delayl }}=2.5 \mathrm{~T}, \tau_{\text {delay } 2}=3.0 \mathrm{~T}$, the temporal amplitudes of laser field from the $2.5 \mathrm{~T}$ to the $3.0 \mathrm{~T}$; from the $3.0 \mathrm{~T}$ to the $3.5 \mathrm{~T}$ are increased and decreased. Figs. 11(c) and 11(d) show the distributions of the MHHG under the above two combined fields. For the case of $\tau_{\text {delay } 1}=2.0 \mathrm{~T}, \tau_{\text {delay } 2}=2.5 \mathrm{~T}$ [Fig. 11(c)], although the 
harmonic cutoff can be remarkably extended, the intensity of the harmonic plateau is reduced. For the case of $\tau_{\text {delay } 1}=2.5 \mathrm{~T}, \tau_{\text {delay } 2}=3.0 \mathrm{~T}$ [Fig. $11(\mathrm{~d})$ ], not only the maximum harmonic cutoff is extended, but also the first harmonic cutoff is decreased, thus leading to the remarkable extension of the harmonic plateau compared with the single $800 \mathrm{~nm}$ field.

Time-frequency analyses of the MHHG for the above two cases are shown in Fig. 12. For the case of $\tau_{\text {delay } 1}=2.0 \mathrm{~T}, \tau_{\text {delay } 2}=2.5 \mathrm{~T}$ [Figs. 12(a) and 12(b)], due to the enhancement of the temporal amplitude from $2.5 \mathrm{~T}$ to the $3.0 \mathrm{~T}$, the recombination energy of the harmonic emission event $A_{2}$ is enhanced. However, due to the decrease of the temporal amplitude from $2.0 \mathrm{~T}$ to the $2.5 \mathrm{~T}$, the ionization rate during this harmonic emission process is very weak, thus leading to the weaker intensity of the harmonic emission event $A_{2}$. For the case of $\tau_{\text {delay } 1}=2.5 \mathrm{~T}, \tau_{\text {delay } 2}=3.0 \mathrm{~T}$ [Figs. $12(\mathrm{c})$ and $\left.12(\mathrm{~d})\right]$, we see that the recombination energies of harmonic emission events $A_{2}$ and $A_{3}$ are enhanced and reduced, respectively, which is responsible for the extension of the maximum harmonic cutoff and the harmonic plateau.

\subsection{Isolated attosecond pulse generation}

Fig. 13 shows the IAP generated through the Fourier transformation of the spectral continuum from $\mathrm{H}_{2}{ }^{+}$driven by the $5 \mathrm{fs} / 800 \mathrm{~nm}$ field combined with two half-cycle controlling pulses with $\tau_{\text {delayl }}=2.5 \mathrm{~T}, \tau_{\text {delay } 2}=3.0 \mathrm{~T}$. Firstly, through analyzing the time-frequency analyses of the MHHG shown in Figs. 12(c) and (d), we see that when the harmonic cutoffs are higher than $75 \omega_{1}$, there is only one harmonic emission event $\left(A_{2}\right)$ 
responsible for the MHHG spectrum. Moreover, the short and the long quantum paths merge in one around the harmonic cutoff region, which is much better for IAP generation [39]. Therefore, by superposing the harmonic cutoff region from the $75 \omega_{1}$ to the $100 \omega_{1}$ orders, an IAP with the full width at half maximum (FWHM) of 48 as can be obtained.

\section{CONCLUSION}

In conclusion, we theoretically study the laser control on the MHHG and the IAP generation from $\mathrm{H}_{2}^{+}$. Laser intensity investigation shows that the contribution from negative- $\mathrm{H}$ to the $\mathrm{MHHG}$ is higher than that from the positive- $\mathrm{H}$ in the middle laser intensity (i.e. $I=4.0 \times 10^{14} \mathrm{~W} / \mathrm{cm}^{2}$ ). However, with the decrease (i.e. $I=2.0 \times 10^{14} \mathrm{~W} / \mathrm{cm}^{2}$ ) or the increase (i.e. $I=7.0 \times 10^{14} \mathrm{~W} / \mathrm{cm}^{2}$ ) of the laser intensity, the asymmetric contributions from the two $\mathrm{H}$ nuclei to the MHHG are decreased. Pulse duration investigation shows that with the increase in laser pulse duration, the asymmetric distributions of the MHHG in two $\mathrm{H}$ are reduced. Laser phase investigation shows that when the laser phase is chosen from $0.0 \pi$ to $0.6 \pi$ and from $1.7 \pi$ to $2.0 \pi$, the contribution from the negative-H plays the main role in MHHG. When the laser phase is chosen from $0.7 \pi$ to the $1.6 \pi$, the contribution from the positive-H to the MHHG is remarkably enhanced and becomes greater than that from the negative- $\mathrm{H}$. The time-frequency analyses of the MHHG and the time-dependent wave function show that the electron is more localized on the negative- $\mathrm{H}$ when the laser field is larger than zero $(E(t)>0.0)$; and the electron prefers to localize on the positive- $\mathrm{H}$ when the laser field changes its direction $(E(t)<0.0)$. Through analyzing the Coulomb potential, we see that the dressed Coulomb 
potential caused by the temporal profile of laser field is the main reason behind the electronic transfer between the two H nuclei. Further, with the introduction of one or two half-cycle controlling pulses, the cutoff and the intensity of the MHHG can be controlled. Finally, by properly selecting the harmonics, an IAP with FWHM of 48 as can be obtained.

\section{ACKNOWLEDGMENTS}

This work was supported by the National Natural Science Foundation of China (No. 11504151), the Doctoral Scientific Research Foundation of Liaoning Province (No. 201501123) and the Scientific Research Foundation of Liaoning Provincial Education Department (Nos. L2014242 and JL201615405).

\section{REFERENCES}

[1] F. Krausz and M. Ivanov, Rev. Mod. Phys. 81, 163 (2009).

[2] T. Seideman, M. Ivanov and P. B. Corkum, Phys. Rev. Lett. 75, 2819 (1995).

[3] L. J. Frasinski, J. H. Posthumus, J. Plumridge, K. Codling, P. F. Taday and A. J. Langley, Phys. Rev. Lett. 83, 3625 (1999).

[4] H. Niikura, P. B. Corkum and D. M. Villeneuve, Phys. Rev. Lett. 90, 203601 (2003).

[5] Y. J. Chen and B. Zhang, Phys. Rev. A 86, 023415 (2012).

[6] I. Yavuz, M. F. Ciappina, A. Chacón, Z. Altun, M. F. Kling and M. Lewenstein, Phys. Rev. A 93, 033404 (2016).

[7] T. Zuo, S. Chelkowski and A. D. Bandrauk, Phys. Rev. A 48, 3837 (1993). 
[8] T. Zuo and A. D. Bandrauk, Phys. Rev. A 52, R2511 (1995).

[9] S. Chelkowski, C. Foisy and A. D. Bandrauk, Phys. Rev. A 57, 1176 (1998).

[10] B. D. Esry, A. M. Sayler, P. Q. Wang, K. D. Carnes and I. B. Itzhak, Phys. Rev. Lett. 97, 013003 (2006).

[11] J. H. Posthumus, Rep. Prog. Phys. 67, 623 (2004).

[12] L. Q. Feng, T. S. Chu and W. Li, Chin. Phys. B, 22, 023302 (2013).

[13] M. Lein, Phys. Rev. Lett. 94, 053004 (2005).

[14] L. Q. Feng and T. S. Chu, J. Chem. Phys. 136, 054102 (2012).

[15] C. Yu, H. X. He, Y. H. Wang, Q. Shi, Y. D. Zhang and R. F. Lu, J. Phys. B: At. Mol. Opt. Phys. 47, 055601 (2014).

[16] K. J. Yuan and A. D. Bandrauk, Phys. Rev. Lett. 110, 023003 (2013).

[17] K. J. Yuan and A. D. Bandrauk, Phys. Rev. A 84, 023410 (2011).

[18] X. B. Bian and A. D. Bandrauk, Phys. Rev. Lett. 105, 093903 (2010).

[19] X. Y. Miao and C. P. Zhao, Laser Phys. Lett. 11, 115301 (2014).

[20] L. Q. Feng and H. Liu, J. Mol. Model 21, 43 (2015).

[21] Y. J. Chen and B. Zhang, Phys. Rev. A 84, 053402 (2011).

[22] P. F. Lan, P. X. Lu, W. Cao, Y. H. Li and X. L. Wang, Phys. Rev. A 76, 021801 (2007).

[23] X. Liu, X. S. Zhu, L. Li, Y. Li, Q. B. Zhang, P. F. Lan and P. X. Lu, Phys. Rev. A 94, 033410 (2016).

[24] I. Yavuz, Y. Tikman, and Z. Altun, Phys. Rev. A 92, 023413 (2015).

[25] L. Q. Feng, Phys. Rev. A 92, 053832 (2015). 
[26] L. Q. Feng, Mol. Phys. 114, 2217 (2016).

[27] P. B. Corkum, Phys. Rev. Lett. 71, 1994 (1993).

[28] Y. N. Pei and X. Y. Miao, Chin. Phys. Lett. 31, 104202 (2014).

[29] J. Zhang, X. L. Ge, T. Wang, T. T. Xu, J. Guo and X. S. Liu, Phys. Rev. A 92, $013418(2015)$.

[30] J. Zhang, X. F. Pan, C. L. Xia, D. Du, T. T. Xu, J. Guo and X. S. Liu, Laser Phys. Lett. 13, 075302 (2016).

[31] J. Zhang, H. F. Liu, X. F. Pan, H. Du, J. Guo and X. S. Liu, Chin. Phys. B 25, $053202(2016)$.

[32] H. Du, X. F. Pan, H. F. Liu, H. D. Zhang, J. Zhang, J. Guo and X. S. Liu, Chin. Phys. B 25, 093202 (2016).

[33] R. F. Lu, P. Y. Zhang and K. L. Han, Phys. Rev. E 77, 066701 (2008).

[34] L. Q. Feng and T. S. Chu, J. Mol. Model 18, 5097 (2012).

[35] J. Hu, K. L. Han and G. Z. He, Phys. Rev. Lett. 95, 123001 (2005).

[36] L. Q. Feng and H. Liu, Can. J. Phys. 94, 651 (2016).

[37] K. Burnett, V. C. Reed, J. Cooper and P. L. Knight, Phys. Rev. A 45, 3347 (1992).

[38] P. Antoine, B. Piraux and A. Maquet, Phys. Rev. A 51, R1750 (1995).

[39] Y. Mairesse, A. D. Bohan, L. J. Frasinski, H. Merdji, L. C. Dinu, P. Monchicourt, P. Breger, M. Kovačev, R. Taïeb, B. Carré, H. G. Muller, P. Agostini and P. Salières, Science 302, 1540 (2003). 


\section{Figure Captions}

Fig. 1 Distributions of the MHHG from $\mathrm{H}_{2}{ }^{+}$under the $5 \mathrm{fs} / 800 \mathrm{~nm}$ field. The laser intensities are (a) $I=2.0 \times 10^{14} \mathrm{~W} / \mathrm{cm}^{2}$; (b) $I=4.0 \times 10^{14} \mathrm{~W} / \mathrm{cm}^{2}$; (c) $I=7.0 \times 10^{14} \mathrm{~W} / \mathrm{cm}^{2}$.

Fig. 2 Laser profiles and ionization probabilities (IPs) of the $5 \mathrm{fs} / 800 \mathrm{~nm}$ field with (a) $I$ $=2.0 \times 10^{14} \mathrm{~W} / \mathrm{cm}^{2} ;$ (d) $I=4.0 \times 10^{14} \mathrm{~W} / \mathrm{cm}^{2} ;$ (g) $I=7.0 \times 10^{14} \mathrm{~W} / \mathrm{cm}^{2}$. Time-frequency analyses of the MHHG from $\mathrm{H}_{2}{ }^{+}$for the cases of the (b) and (c) $I=2.0 \times 10^{14} \mathrm{~W} / \mathrm{cm}^{2}$; (e) and (f) $I=4.0 \times 10^{14} \mathrm{~W} / \mathrm{cm}^{2}$; (h) and (i) $I=7.0 \times 10^{14} \mathrm{~W} / \mathrm{cm}^{2}$. Left column means the time-frequency analyses of the MHHG from negative- $\mathrm{H}$ and the right column means the time-frequency analyses of the MHHG from positive-H. T means the optical cycle of $800 \mathrm{~nm}$ pulse.

Fig. 3 Distributions of the MHHG from $\mathrm{H}_{2}{ }^{+}$under the $10 \mathrm{fs} / 800 \mathrm{~nm}$ field with $I=$ $4.0 \times 10^{14} \mathrm{~W} / \mathrm{cm}^{2}$.

Fig. 4 (a) Laser profile of the $10 \mathrm{fs} / 800 \mathrm{~nm}$ field. Time-frequency analyses of the MHHG from $\mathrm{H}_{2}{ }^{+}$driven by the above $10 \mathrm{fs} / 800 \mathrm{~nm}$ field (b) negative- $\mathrm{H}$ and (c) positive- $\mathrm{H}$.

Fig. 5 (a) Laser phase effect on the intensities of the MHHG spectra from the two H nuclei. (b) Intensities differences of the MHHG spectra from the two H nuclei. The laser field is $5 \mathrm{fs} / 800 \mathrm{~nm}, I=4.0 \times 10^{14} \mathrm{~W} / \mathrm{cm}^{2}$.

Fig. 6 Laser profiles of the above $5 \mathrm{fs} / 800 \mathrm{~nm}$ with (a) $\varphi=0.5 \pi$; and (d) $\varphi=1.0 \pi$. Time-frequency analyses of the MHHG for the cases of (b) $\varphi=0.5 \pi, \mathrm{z}<0.0$ a.u.; (c) $\varphi=$ $0.5 \pi, \mathrm{z}>0.0$ a.u. and (e) $\varphi=1.0 \pi, \mathrm{z}<0.0$ a.u.; (f) $\varphi=1.0 \pi, \mathrm{z}>0.0$ a.u..

Fig. 7 Time-dependent wave function $|\psi(z, R, t)|^{2}$ of $\mathrm{H}_{2}^{+}$with (a) $t=2.0 \mathrm{~T}$; (b) $t=2.5 \mathrm{~T}$; (c) $t=3.0 \mathrm{~T}$; (d) $t=3.5 \mathrm{~T}$. The laser field is $5 \mathrm{fs} / 800 \mathrm{~nm}$ field with $I=4.0 \times 10^{14} \mathrm{~W} / \mathrm{cm}^{2}, \varphi=$ $0.0 \pi$.

Fig. 8 The energy combined Coulomb potential and laser field potential of $\mathrm{H}_{2}{ }^{+}$for the case of $5 \mathrm{fs} / 800 \mathrm{~nm}$ field with $I=4.0 \times 10^{14} \mathrm{~W} / \mathrm{cm}^{2}, \varphi=0.0 \pi$.

Fig. 9 Laser profiles of the single-color $5 \mathrm{fs} / 800 \mathrm{~nm}$ field, the half-cycle controlling fields and the combined fields with (a) $\tau_{\text {delay }}=2.0 \mathrm{~T}$; (b) $\tau_{\text {delay }}=2.5 \mathrm{~T}$; (c) $\tau_{\text {delay }}=3.0 \mathrm{~T}$. The intensity and the pulse duration of the half-cycle controlling pulse is $k E=0.3 E, \tau_{\mathrm{HCP}}=$ 2.0 fs. Distributions of the MHHG driven by the above three combined fields with (d) 
$\tau_{\text {delay }}=2.0 \mathrm{~T} ;$ (e) $\tau_{\text {delay }}=2.5 \mathrm{~T}$; (f) $\tau_{\text {delay }}=3.0 \mathrm{~T}$.

Fig. 10 Time-frequency analyses of the MHHG for the cases of the combined fields with (a) and (b) $\tau_{\text {delay }}=2.0 \mathrm{~T}$; (c) and (d) $\tau_{\text {delay }}=2.5 \mathrm{~T}$; (e) and (f) $\tau_{\text {delay }}=3.0 \mathrm{~T}$. Left column means the time-frequency analyses of the MHHG from negative- $\mathrm{H}$ and the right column means the time-frequency analyses of the MHHG from positive-H.

Fig. 11 Laser profiles of the single-color $5 \mathrm{fs} / 800 \mathrm{~nm}$ field, the two half-cycle controlling fields and the combined fields with (a) $\tau_{\text {delay } 1}=2.0 \mathrm{~T}$, $\tau_{\text {delay } 2}=2.5 \mathrm{~T}$; (b) $\tau_{\text {delay } 1}=2.5 \mathrm{~T}, \tau_{\text {delay } 2}$ $=3.0 \mathrm{~T}$. Distributions of the MHHG driven by the above two combined fields with (c) $\tau_{\text {delay } 1}=2.0 \mathrm{~T}, \tau_{\text {delay } 2}=2.5 \mathrm{~T} ;$ (d) $\tau_{\text {delay } 1}=2.5 \mathrm{~T}, \tau_{\text {delay } 2}=3.0 \mathrm{~T}$.

Fig. 12 Time-frequency analyses of the MHHG for the cases of the combined fields with (a) and (b) $\tau_{\text {delay } 1}=2.0 \mathrm{~T}, \tau_{\text {delay } 2}=2.5 \mathrm{~T}$; (c) and (d) $\tau_{\text {delay } 1}=2.5 \mathrm{~T}, \tau_{\text {delay } 2}=3.0 \mathrm{~T}$. Left column means the time-frequency analyses of the MHHG from negative- $\mathrm{H}$ and the right column means the time-frequency analyses of the MHHG from positive- $\mathrm{H}$.

Fig. 13 Temporal profile of the IAP by superposing the harmonics of the combined field with $\tau_{\text {delay } 1}=2.5 \mathrm{~T}, \tau_{\text {delay } 2}=3.0 \mathrm{~T}$ from the $75 \omega_{1}$ to the $100 \omega_{1}$. 


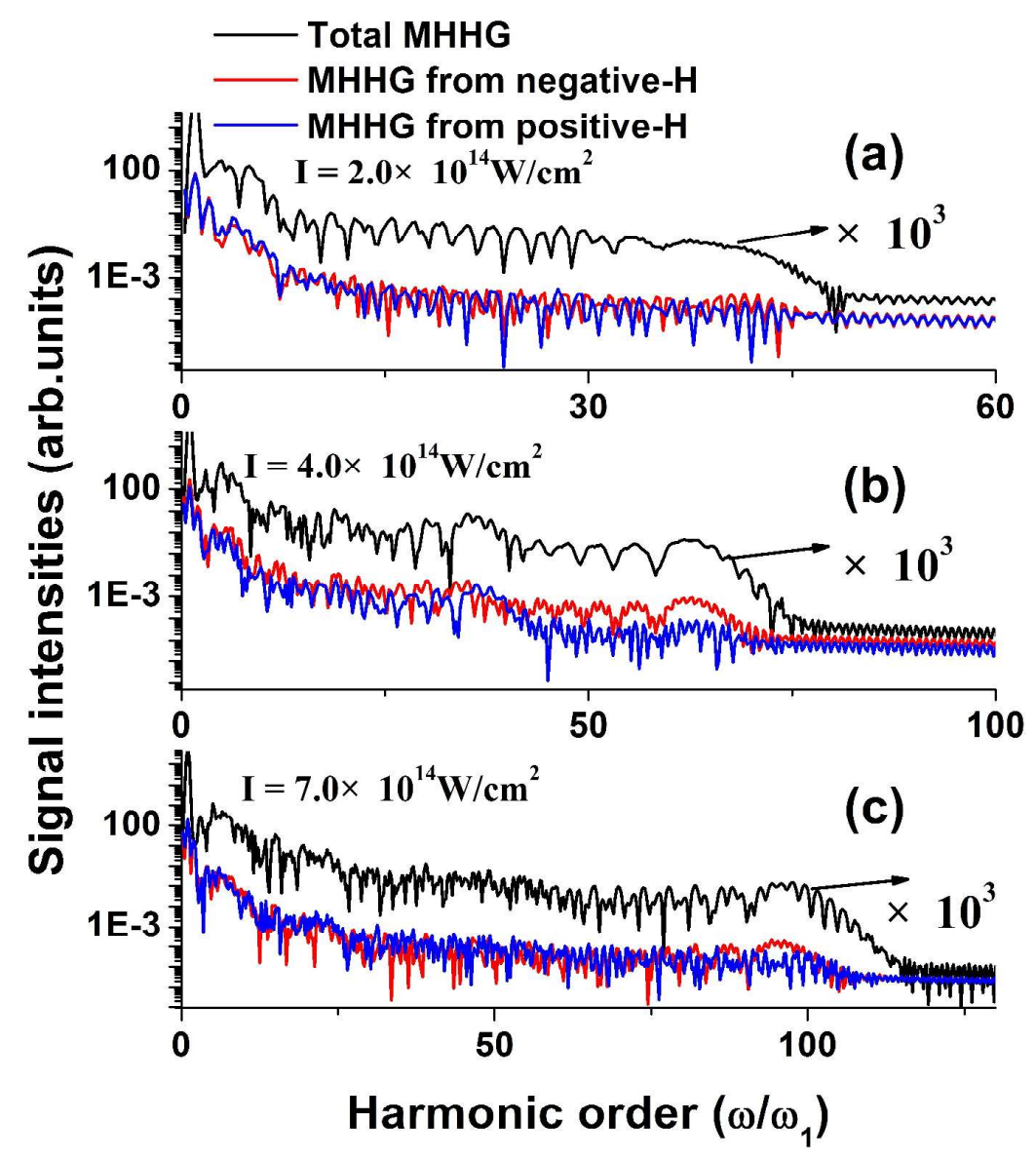

Figure 1 

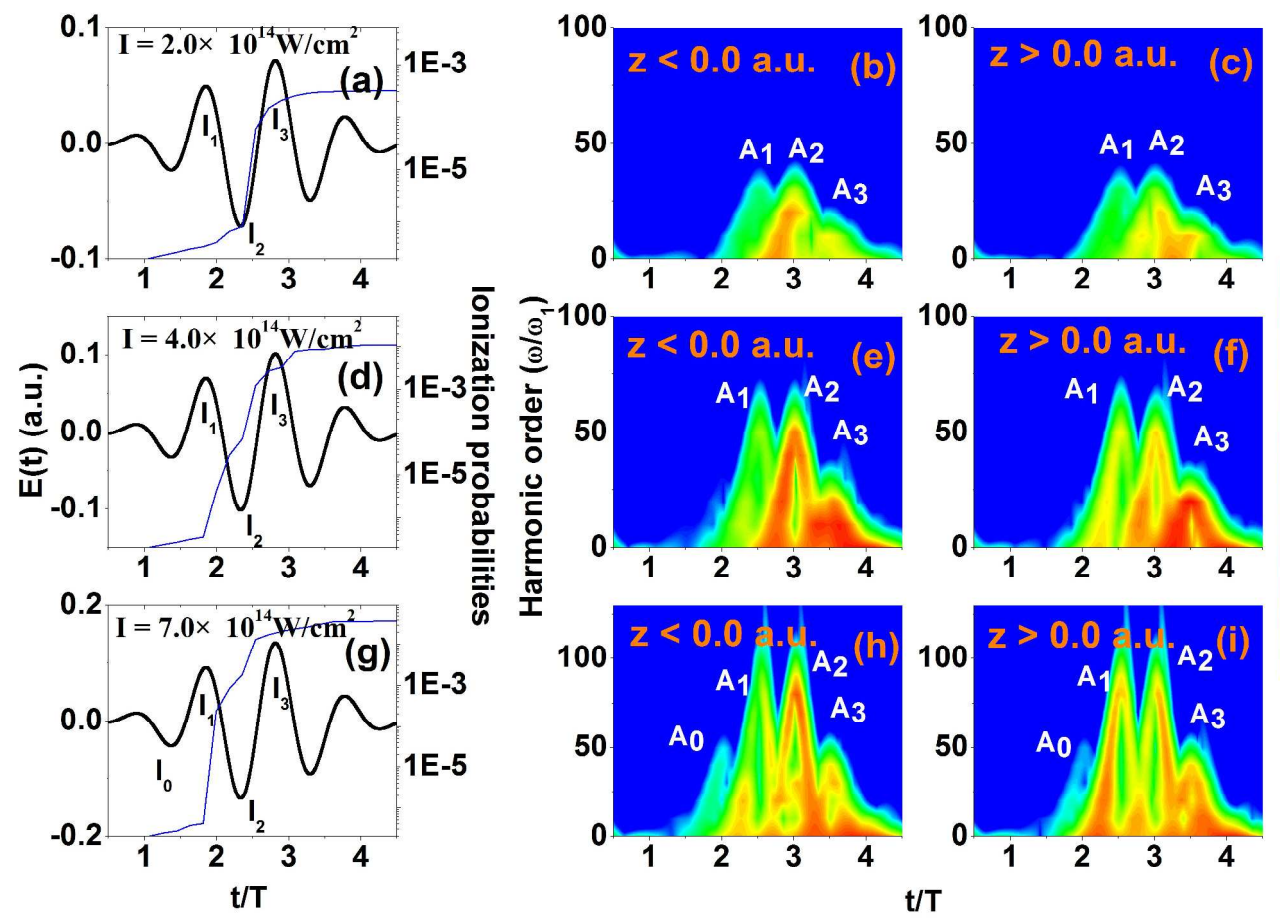

-2.000
-3.000
-4.000
-5.000
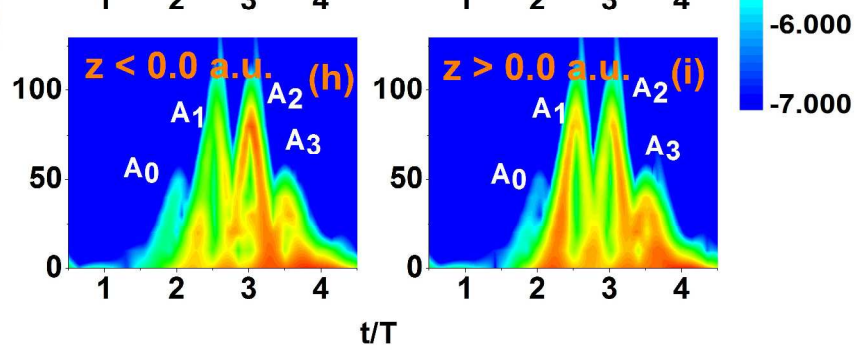

Figure 2 


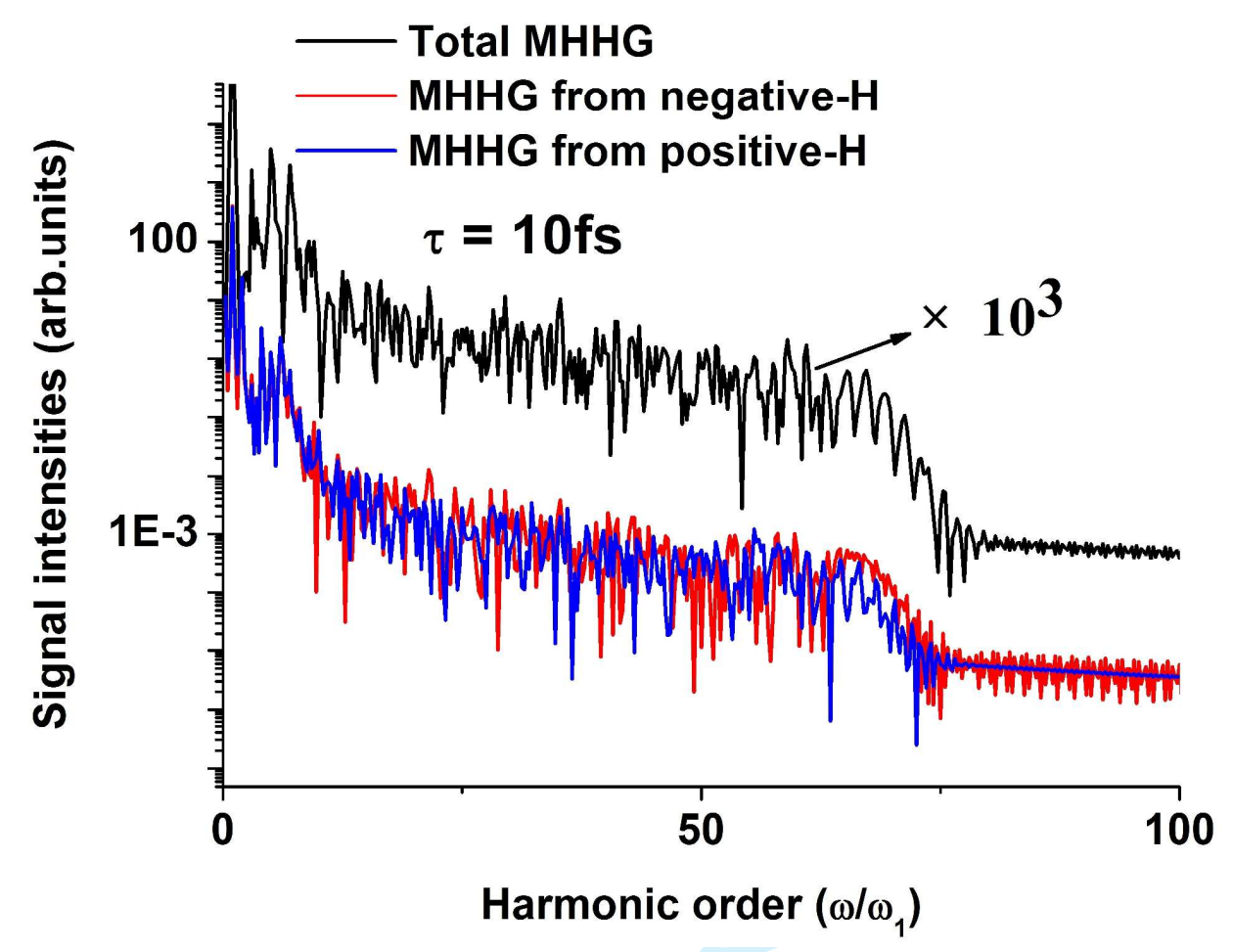

Figure 3 


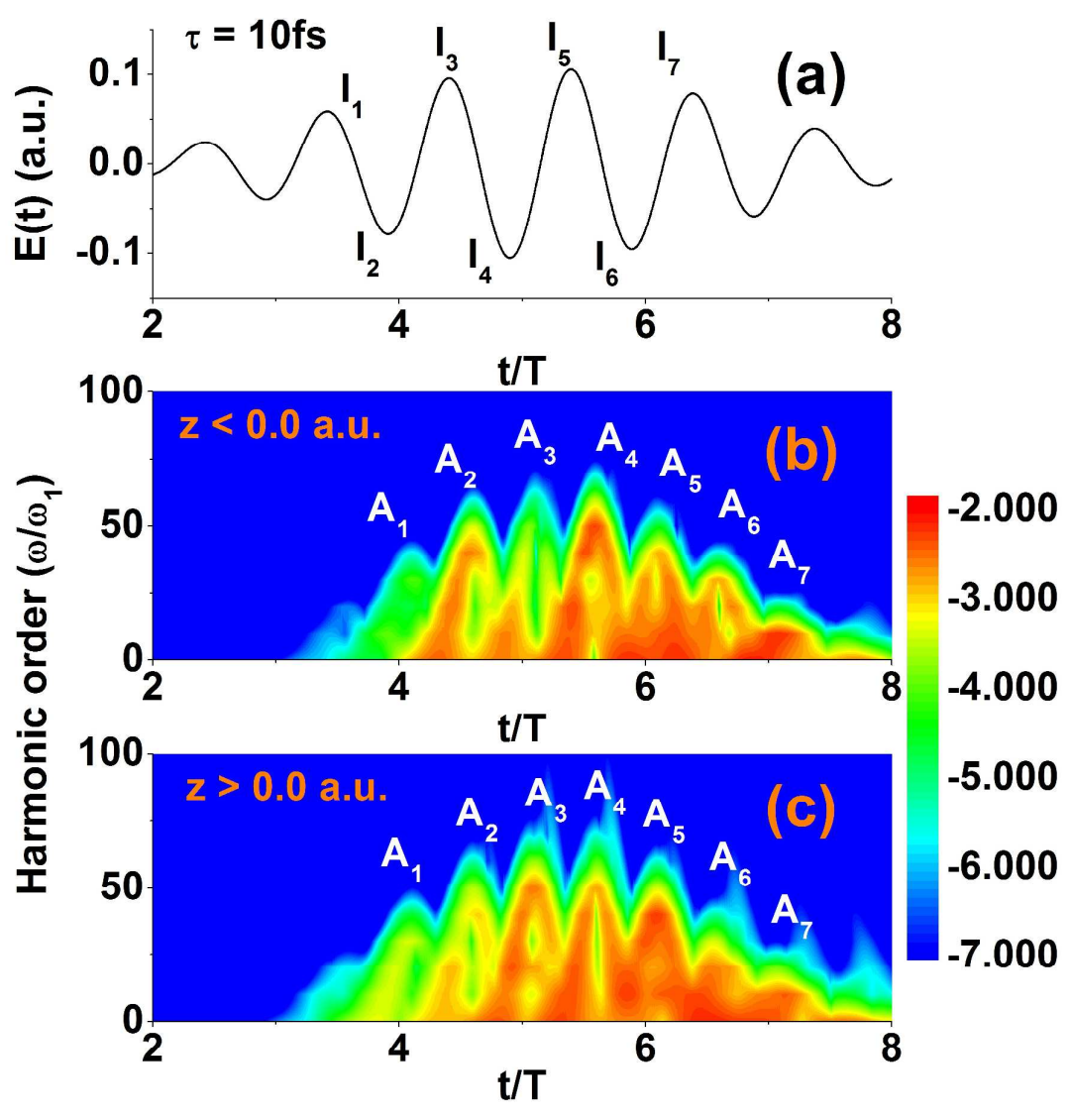

Figure 4 

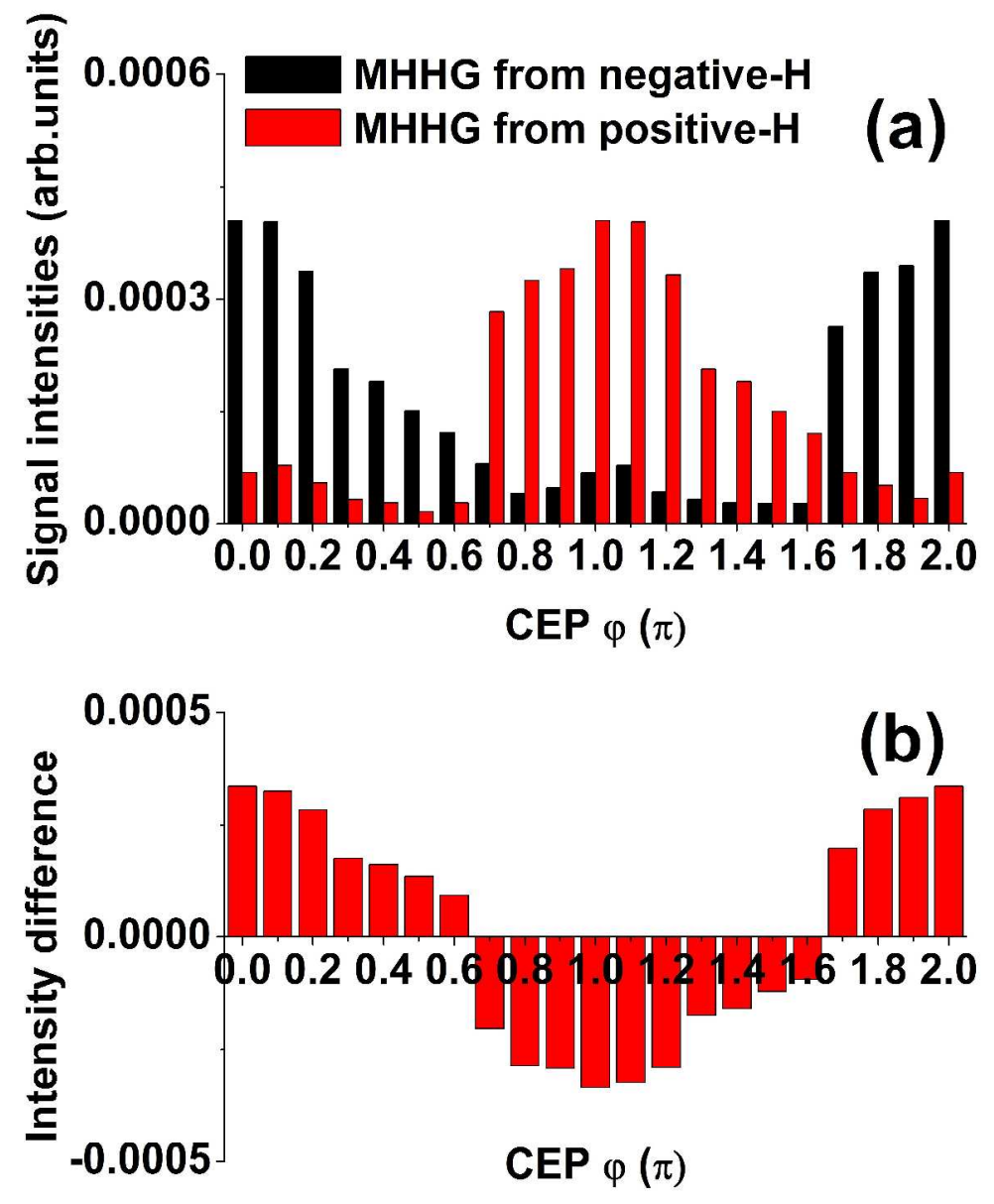

Figure 5 

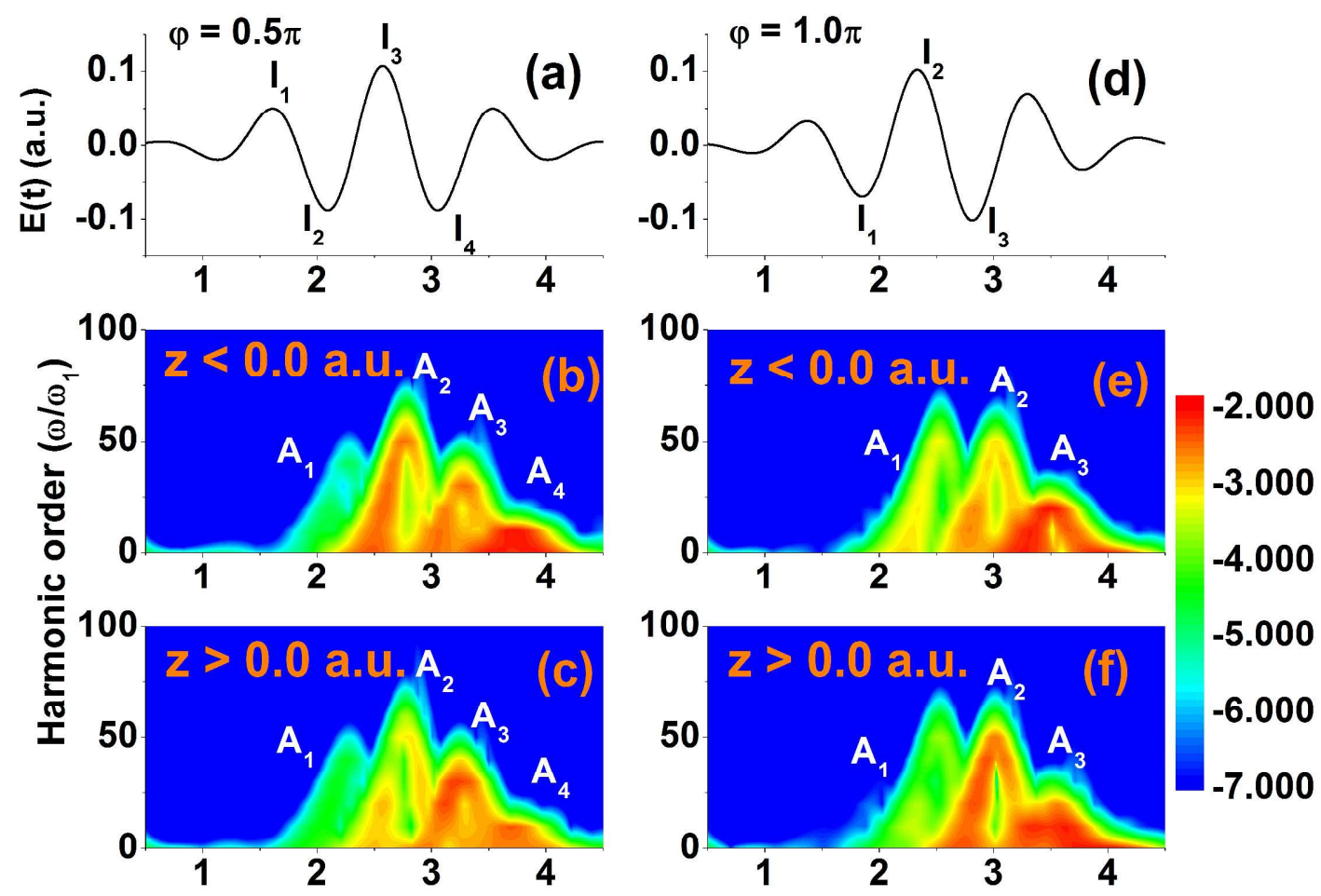

$t / T$

Figure 6 


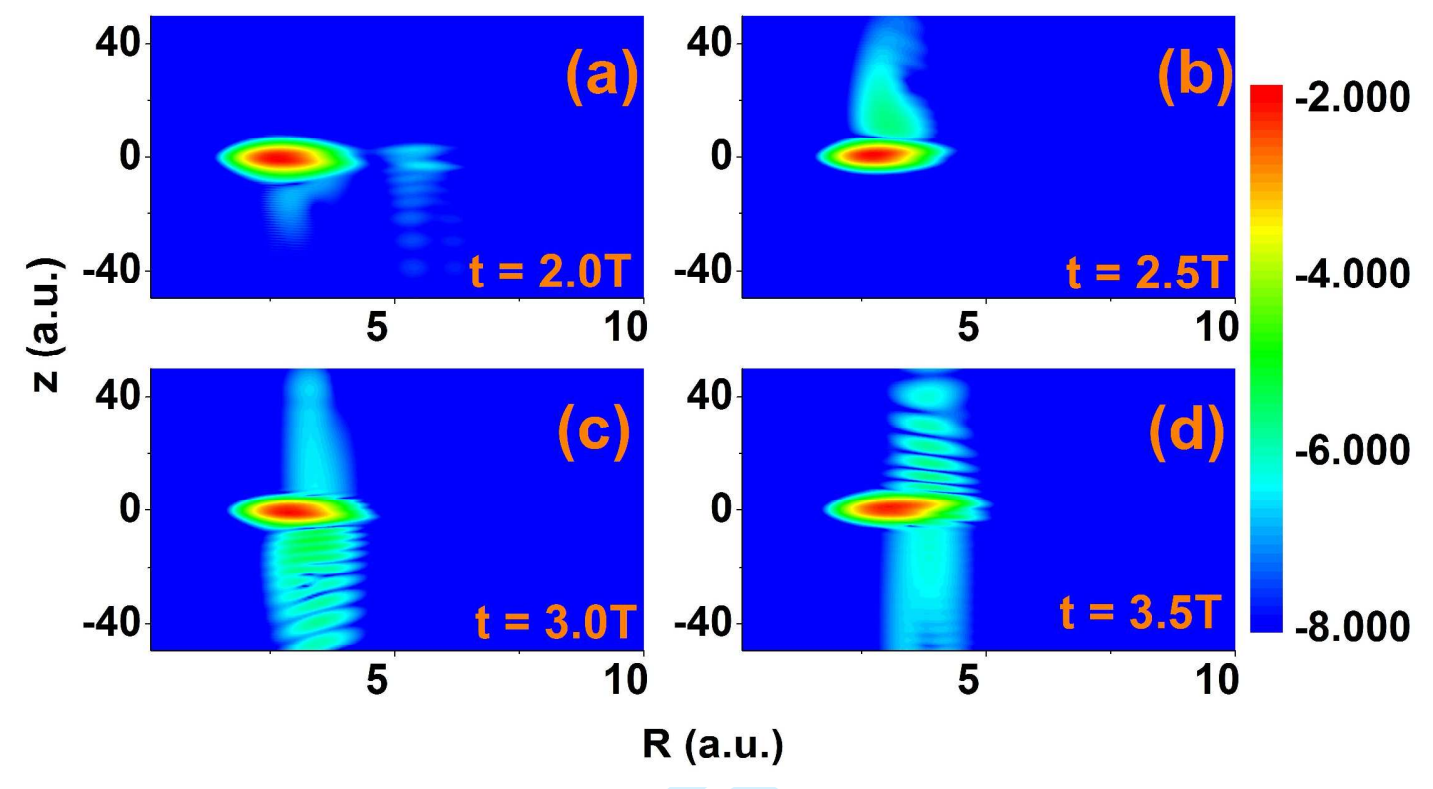

Figure 7 


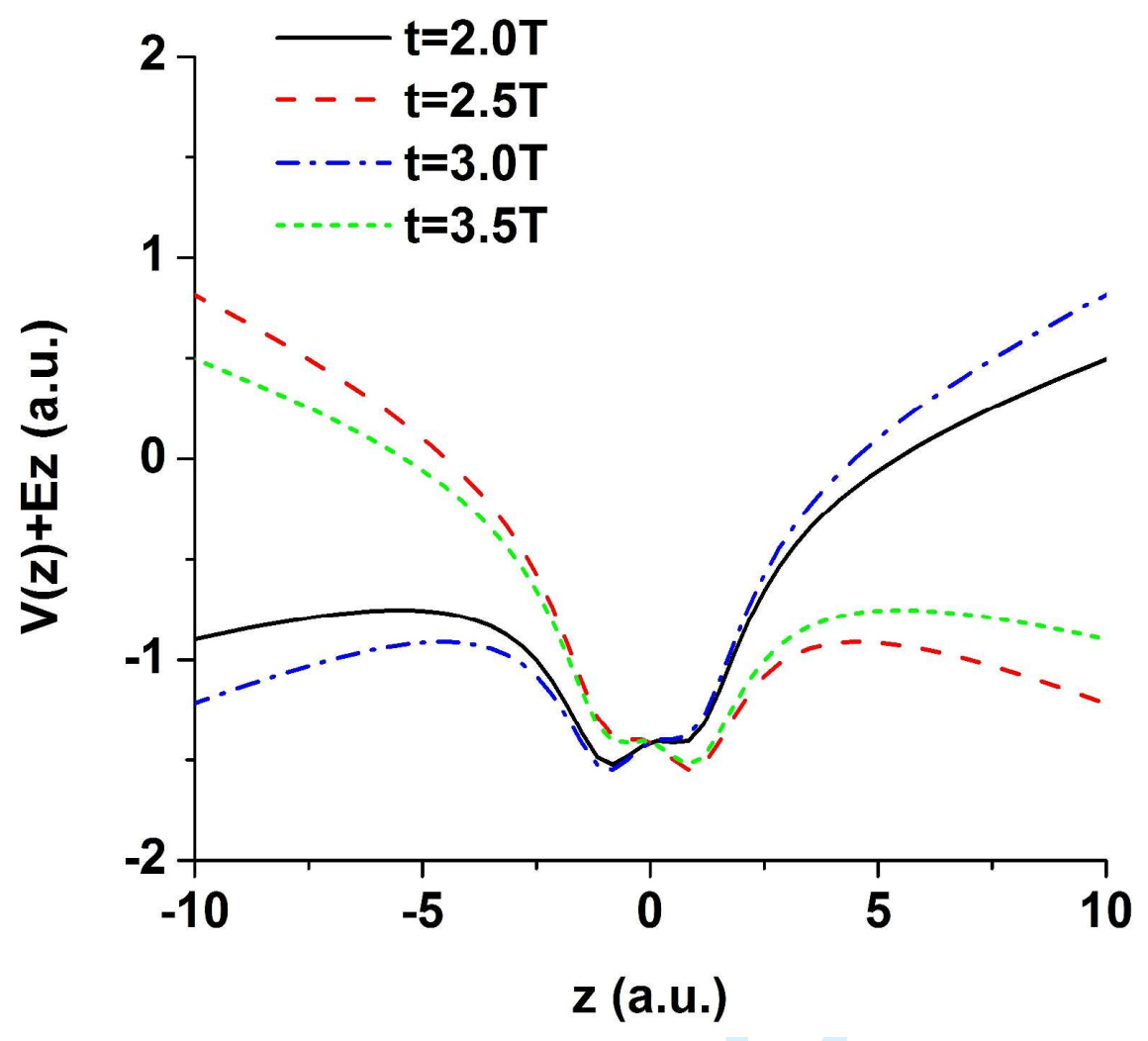

Figure 8 

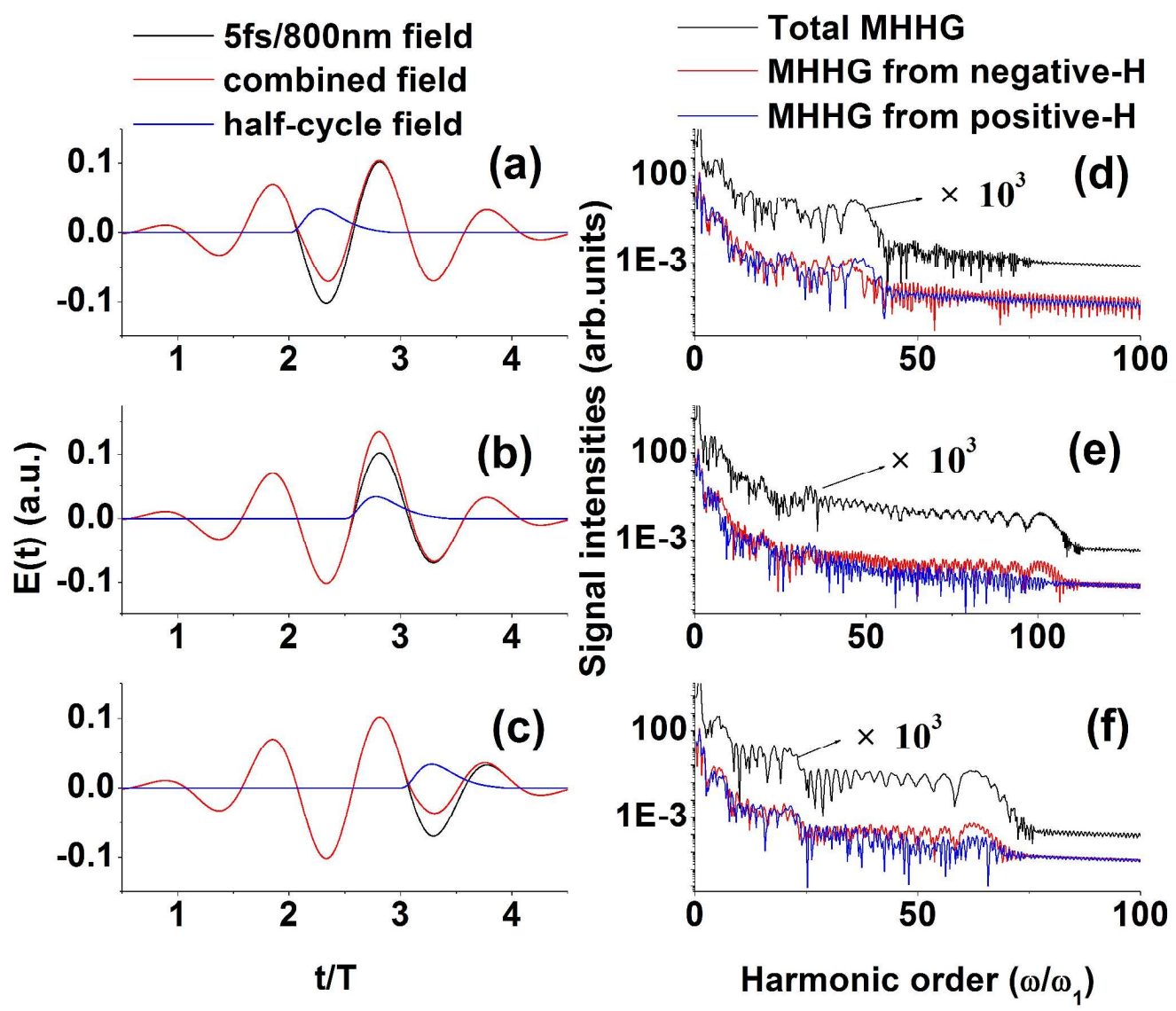

Figure 9 


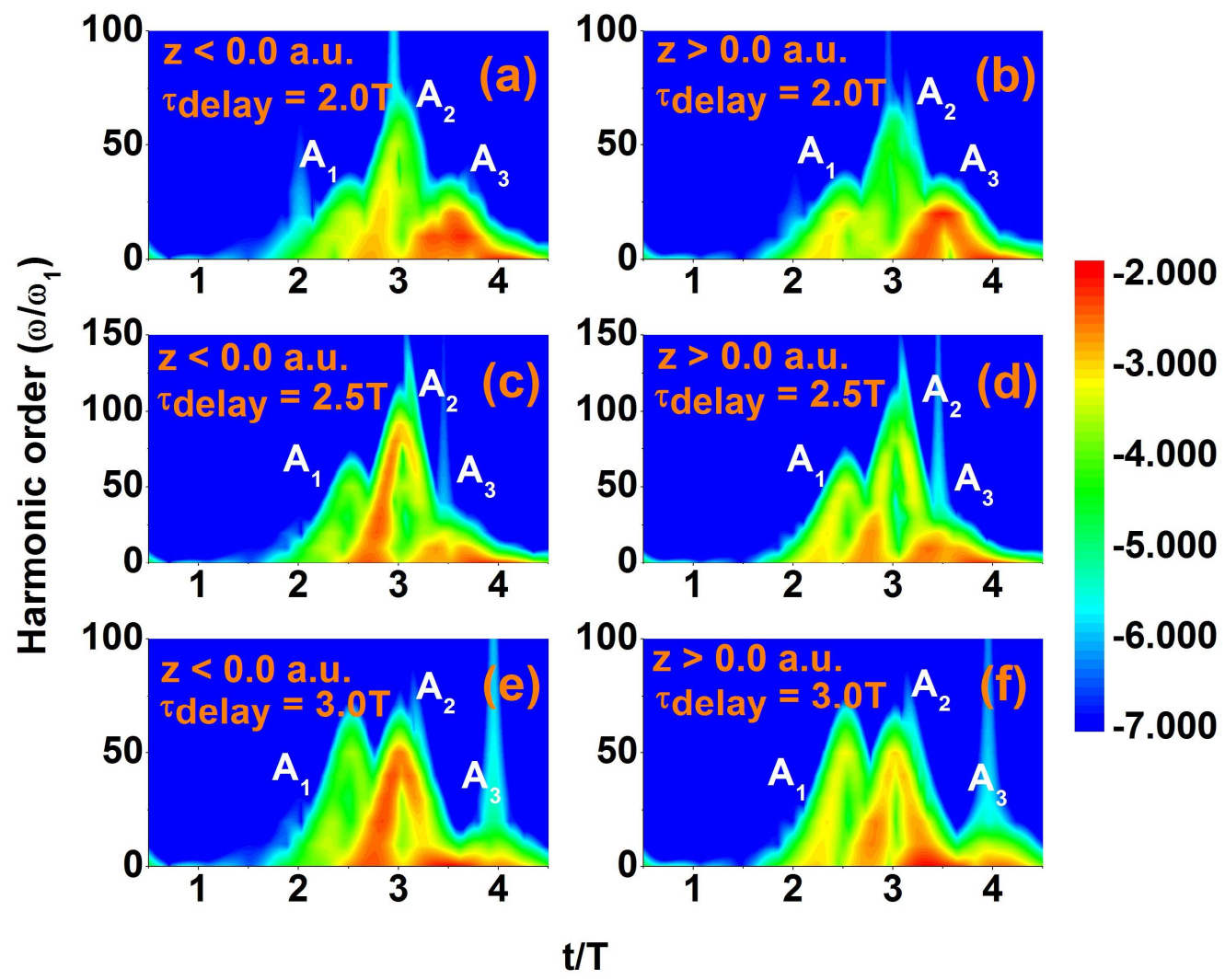

Figure 10 

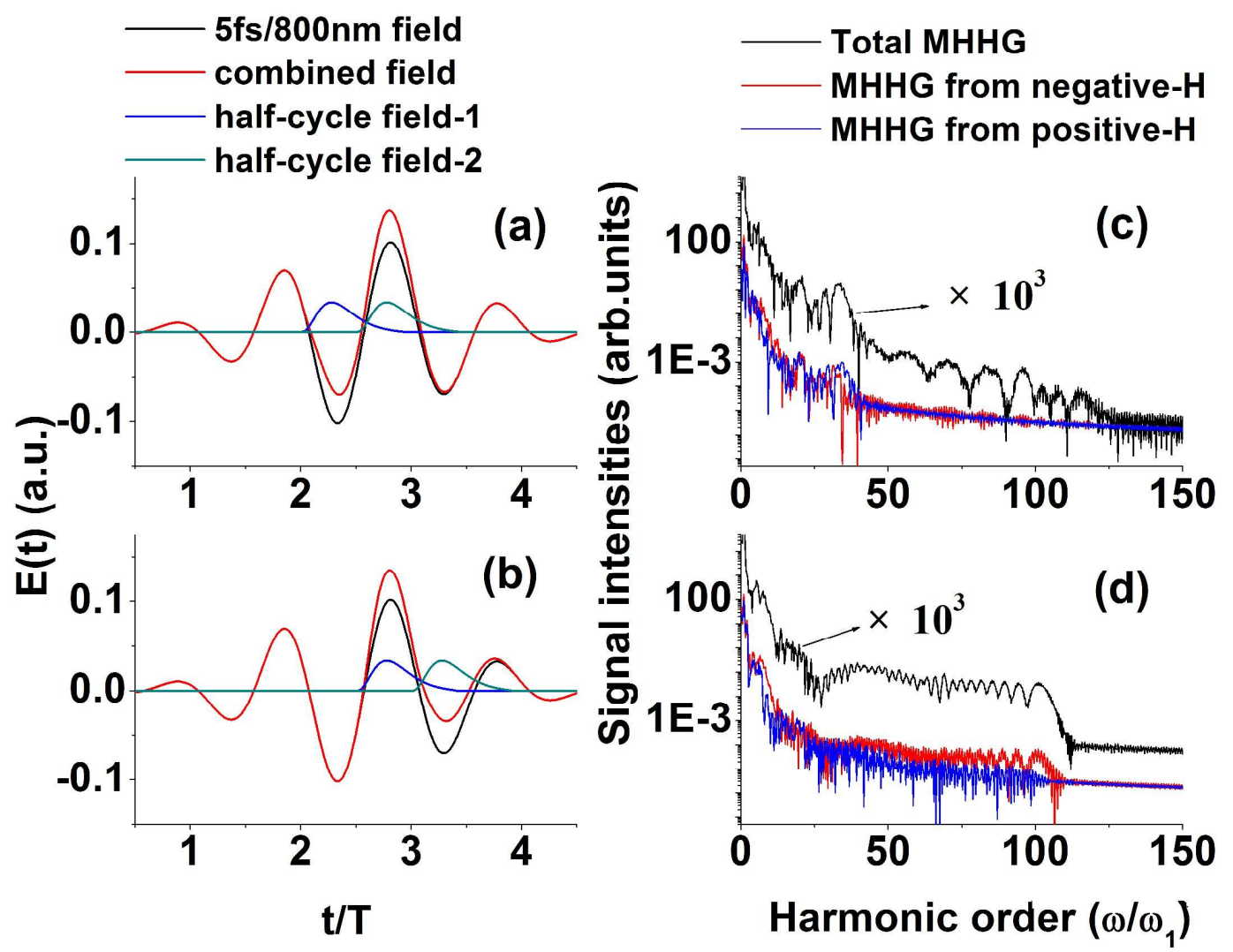

Figure 11 


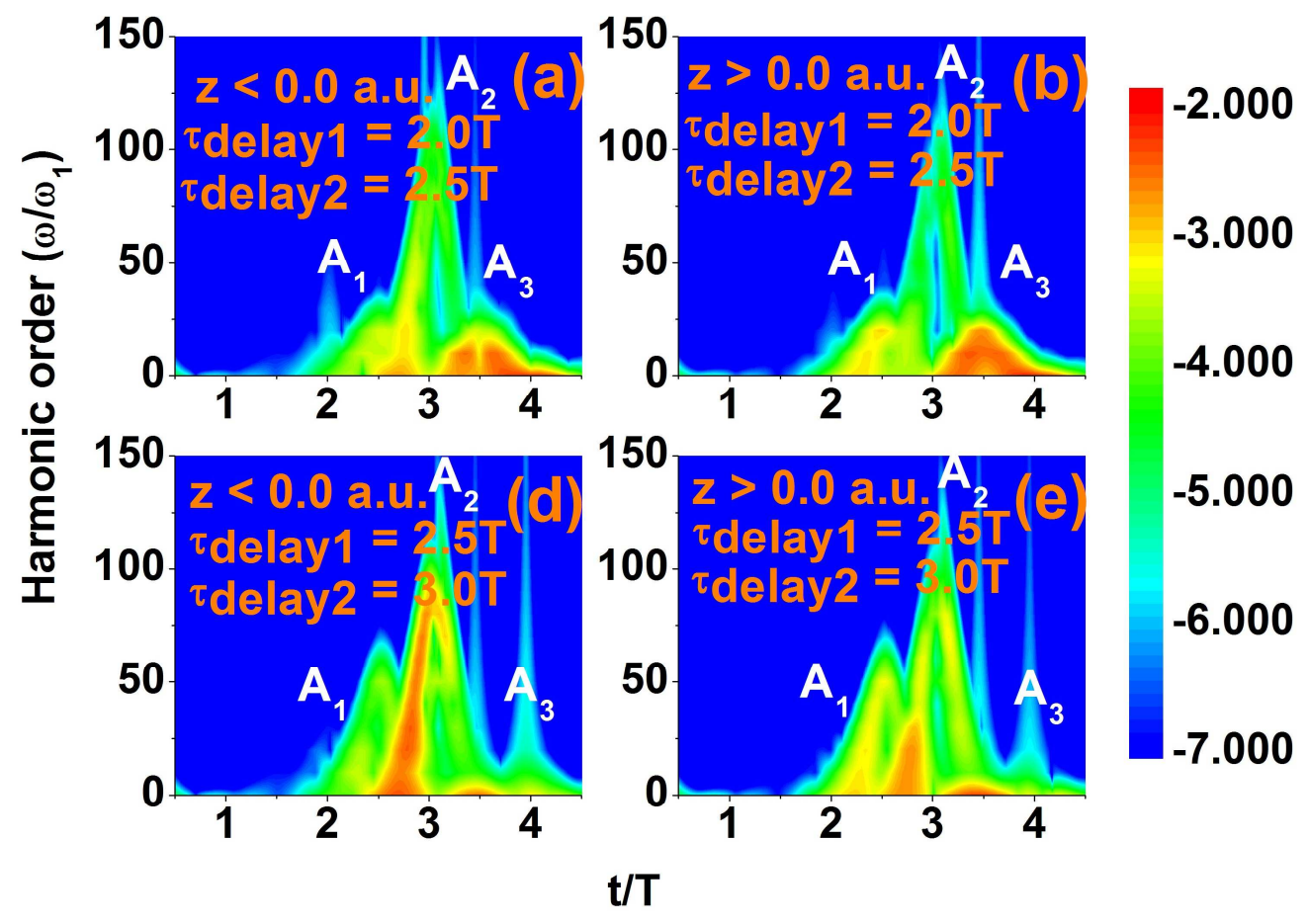

Figure 12 


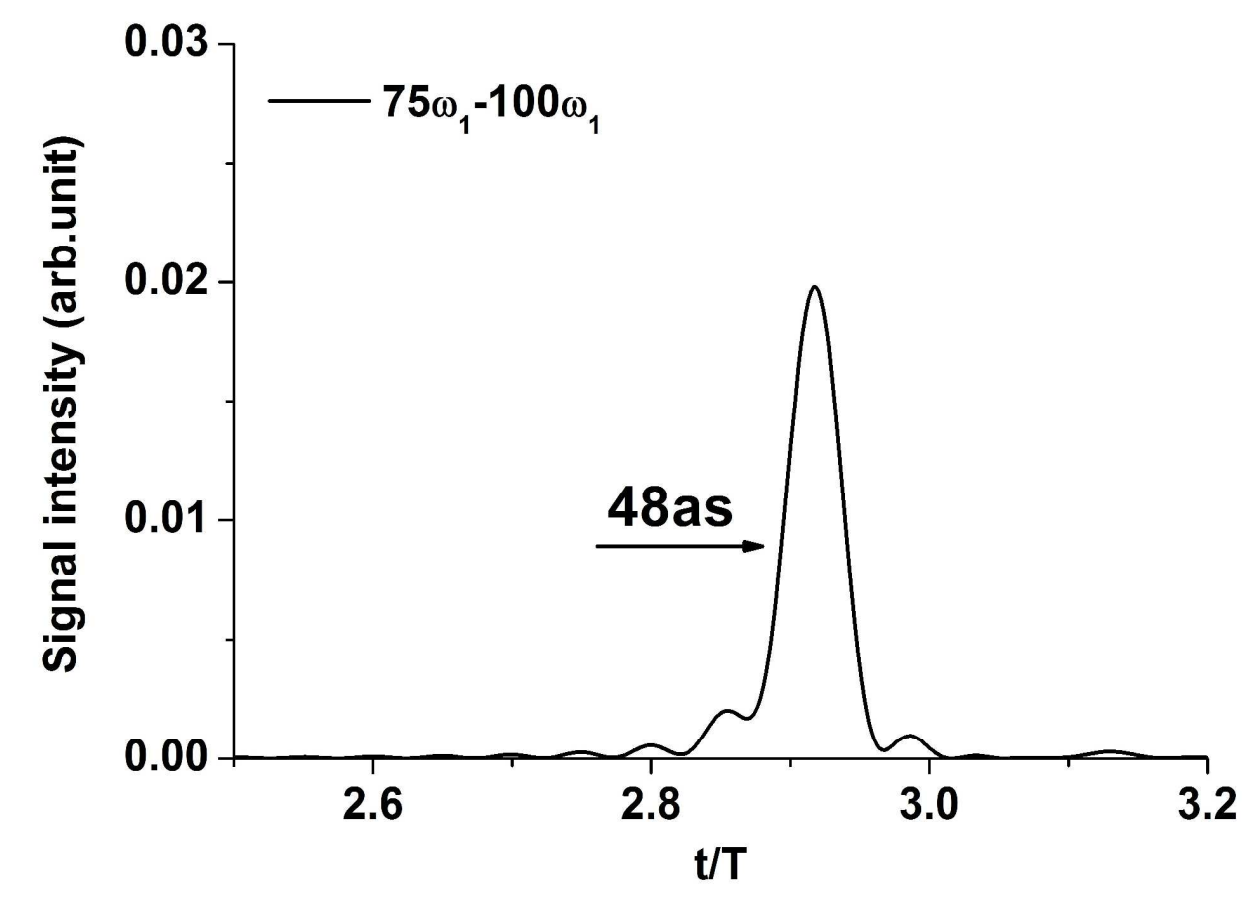

Figure 13 